\title{
Testing for amphetamine-type stimulant (ATS) use to ascertain validity of self-reported ATS use among young female sex workers in Cambodia
}

\author{
Vannda Kab ${ }^{1}$, Jennifer Evans ${ }^{2}$, Neth Sansothy ${ }^{3}$, Ellen Stein ${ }^{2}$, Marie Claude-Couture ${ }^{2}$, Lisa Maher ${ }^{4}$ and \\ Kimberly Page ${ }^{2^{*}}$ on behalf of the Young Women's Study Collaborative
}

\begin{abstract}
Objective: To assess concordance between self-reported amphetamine-type stimulant (ATS) use and toxicology results among young female sex workers (FSW) in Phnom Penh, Cambodia.

Methods: Cross-sectional data from the Young Women's Health Study-2 (YWHS-2), a prospective study of HIV and ATS use among young (15 to 29 years) FSW in Phnom Penh, Cambodia, was analyzed. The YWHS-2 assessed sociodemographic characteristics, HIV serology, HIV risk, and ATS use by self-report and urine toxicology testing at each quarterly visit, the second of which provided data for this assessment. Outcomes include sensitivity, specificity, positive- and negative predictive values (overall and stratified by age), sex-work setting, and HIV status.

Results: Among 200 women, prevalence of positive toxicology screening for ATS use was 14\% (95\% confidence interval [Cl], 9.2, 18.9\%) and concurrent prevalence of self-reported ATS was 15.5\% (95\% Cl, 10.4, 20.6\%). The sensitivity and specificity of self-reported ATS use compared to positive toxicology test results was 89.3\% (25/28), and $96.5 \%$ (166/172), respectively. The positive predictive value of self-reported ATS use was $80.6 \%(25 / 31)$; the negative predictive value was $98.2 \%$ (166/169). Some differences in concordance between self-report and urine toxicology results were noted in analyses stratified by age group and sex-work setting but not by HIV status.

Conclusion: Results indicate a high prevalence of ATS use among FSW in Phnom Penh, Cambodia, and high concordance between self-reported and toxicology-test confirmed ATS use.
\end{abstract}

\section{Introduction}

Cambodia has the highest HIV prevalence of any Asian country, and over the last decade has experienced the most serious HIV/AIDS epidemic in Southeast Asia [1]. Heterosexual contact is the major route of HIV transmission, and female sex workers (FSW) remain the group at highest risk [2]. Although crucial progress has been made in reducing risky sexual behavior, including widespread condom use and promotion of reduced number of sexual partners, HIV prevalence among FSW remains high, ranging from $11 \%$ to $26 \%$ [2-5]. Poverty [6], low literacy [7], a high prevalence of sexually transmitted infections (STI) [4], and a highly mobile workforce [8] are contributing factors to the epidemic.

\footnotetext{
* Correspondence: Kpage@psg.ucsf.edu

${ }^{2}$ Department of Epidemiology and Biostatistics, University of California San

Francisco, 50 Beale Street, Ste 1200, San Francisco, CA 94105, USA

Full list of author information is available at the end of the article
}

Recent research has also identified drug use and, in particular, amphetamine-type stimulant (ATS) use as a serious emerging problem associated with HIV risk among FSW $[3,9,10]$, which threatens to reverse downward trends in HIV infection rates in the region.

Amphetamine-type stimulants include a range of synthetic psychostimulants, including methamphetamine, amphetamine, and ecstasy, which can be injected, smoked, or taken orally. Effects of these drugs include euphoria, alertness, arousal, increased libido, increased sympathetic nervous responses, (heart rate, respiratory rate, blood pressure), and perceived increases in confidence, energy and physical strength [11]. In Cambodia, a pill form of methamphetamine known as "yama" is widely produced, trafficked, and used. The tablets generally contain about 25\% methamphetamine. "Crystal" (also known as "ice") is generally about $85 \%$ methamphetamine and more addictive. Although yama pills are 
swallowed, both forms are usually melted and the vapors inhaled, resulting in rapid neurologic effects $[11,12]$. Use of ATS has been associated with elevated HIV risk behavior in many countries and in several population subgroups [3,9,13-19]. The United Nations Office on Drugs and Crime (UNODC) reports that use of these drugs is widespread in Asia and increasing rapidly in Cambodia [20]. In Cambodia, ATS are highly available both in pill and crystalline form and are generally ingested or smoked; injection use is uncommon [21]. The Cambodia National Authority for Combating Drug Abuse (NACD) estimated that $70 \%$ of all drug users in Cambodia use ATS [20]. The drug accounts for the majority of all drug seizures by authorities, and, in pill form, has been ranked as the leading drug of abuse for the past nine years with consistent increases since 2006 [20], at which time it was estimated that 30,000 tablets of yama were consumed orally or smoked there daily. Use is particularly high among vulnerable populations, including FSW $[3,9]$, men who have sex with men (MSM), and street children $[12,20,21]$.

Measuring drug use in epidemiological research studies poses challenges. Self-reported measures of drug use have the advantage of being noninvasive and permit evaluation over longer time periods compared with biochemical assessments [22]. However, study participants may misrepresent drug use due to social desirability bias, stigma, poor recall, poorly worded questions, or poorly worded response categories in surveys and interviews $[23,24]$, all of which could result in misclassification of measured exposures. Although studies have shown that the use of Audio Computer-Assisted Self Interview (ACASI) increases reporting of sensitive and stigmatized behaviors [25,26], research suggests that the validity of self-reported drug use varies by population [27], race/ ethnicity [28-30], mental health [27], and drug treatment status $[22,31,32]$, although not by gender [30,33]. Accuracy has varied in studies of arrestee populations [34,35] but have been reported as higher in groups sampled in emergency department and STI clinics [34]. Those that report more frequent drug use, compared to infrequent use, are more likely to self-report recent drug use $[27,36]$. Urine toxicology assessments provide sensitive and valid measures of many drug types; but some, like ATS, are restricted to a short timeframe due to rapid metabolization. The detection window may also depend on the physical condition of the individual (e.g., degree of hydration), route of drug ingestion (e.g., oral, intranasal, or intravenous), frequency of use, and drug-related factors such as purity [27].

To explore the validity of self-reported ATS use among young FSW in Phnom Penh, Cambodia, we compared self-reported ATS use with results from concurrently collected urine toxicology tests. We also examine whether sociodemographic, sex-work venue, and HIV status were associated with validity of self-reported ATS use.

\section{Methods \\ Study setting}

The Young Women's Health Study (YWHS-2) was a prospective study of HIV and ATS use among 15-29 year-old women engaged in sex work in a diversity of settings in Phnom Penh, Cambodia [10]. Data for this cross-sectional validity assessment was collected at the second quarterly study visit women attended. Both self-reported measures and urine toxicology testing for ATS were assessed. The YWHS-2 was led by HIV-research and HIV-prevention specialists from the Cambodian $\mathrm{Na}$ tional Center for HIV, AIDS, Dermatology, and STDs (NCHADS), the Cambodian Women's Development Association (CWDA), the University of California San Francisco (UCSF) in the United States, and the Kirby Center (formerly National Centre for HIV Epidemiology and Clinical Research) at the University of New South Wales (UNSW) in Australia.

\section{Study participants and data collection}

Young women at high risk of HIV infection were the target study population. Inclusion criteria were age 15-29 years, understanding of spoken Khmer, Cambodian ethnicity, reporting of at least two different sexual partners in the last month or engaging in transactional sex (sex in exchange for money, goods, services, or drugs) within the last three months, plans to stay in the Phnom Penh area for 12 months, being biologically female, and being able to provide voluntary informed consent. Study methods have been described previously [3]. In brief, trained field assistants from the CWDA recruited women from community locations, provided study information, and obtained group informed consent. Women who consented were then seen by appointment at the YWHS-2 clinic site; free transportation was provided. Participants received US \$5 and condoms at each study visit. Contact information was collected to facilitate participant tracking and maximize follow-up. Women were asked to enroll for a one-year study with quarterly study visits.

Data collection for this validity assessment occurred in November 2009 at the second study visit (month 3 of the study). All study visits included administration of a structured questionnaire in Khmer by trained interviewers who queried participants about sociodemographic characteristics, health care, occupational and sexual risk exposures, alcohol and self-reported ATS use as well as testing for HIV and ATS using blood and urine samples, respectively. The second visit (at 3 months) was used for this cross-sectional analysis because questions about past 48-hour drug use (corresponding to when 
urine toxicology screening could detect ATS) were added to the questionnaire starting with that visit; thus, it was the first available time point for comparison and validation of self-reported use and urine testing. Women were asked about type of ATS use (yama, ice, and/or crystal); frequency and route of use (ingestion, smoking or injected) since their last (baseline) visit; number of days of use in the past month; and use in the past five days, with specific questions including "today," "yesterday," and "two," "three," "four," and "five" days ago. Urine toxicology testing (Innovacon Multi-Drug Screen Test Panel Dip, Redwood Toxicology Laboratories, Santa Rosa, CA) was conducted to qualitatively screen for recent ATS, opiate, and cannabis use. Women were asked to void into prelabeled sterile collection cups in a private lavatory; the specimens were passed through a private window to the onsite laboratory for testing. The test included four strips, which yielded positive results for amphetamine and/or methamphetamine if either exceeded $1000 \mathrm{ng} / \mathrm{mL}$; for opiates if morphine in urine exceeded $2000 \mathrm{ng} / \mathrm{mL}$; and for cannabis if the concentration 11-nor- $\Delta 9$-tetrahydrocannabinol-9-carboxylic acid (THC-COOH) exceeded $50 \mathrm{ng} / \mathrm{mL}$. A positive amphetamine or methamphetamine screen (the primary outcome of interest) was considered indicative of ATS use in the past 48 hours. Drug screening was conducted in conjunction with client-centered risk reduction counseling.

\section{Ethical review}

The study protocol was reviewed and approved by the Cambodian National Ethics Committee, the University of California San Francisco Institutional Review Board, and the University of New South Wales Human Research Ethics Committee.

\section{Data analysis}

The primary outcomes of interest were ATS use by selfreport in the past two days and ATS use by urine screening. Correlates of interest included age, HIV status, and sexwork venue. Both self-report and urine-screened ATS coded as dichotomous variables. Descriptive statistics including measures of central means, medians and ranges, and frequencies were assessed for continuous and categorical variables, respectively. Chi-square tests were used to assess differences between proportions, including selfreported past two-day use of ATS and urine toxicology ATS results. Sensitivity, specificity, positive- and negativepredictive values, and respective 95\% confidence intervals (95\% CI) were computed. We conducted sub-analyses stratifying by age, HIV status, and sex work setting. Analyses were performed using STATA/IC 11.1 (STATA, College Station, TX, USA.)

\section{Results}

Two hundred twenty women were enrolled in YWHS-2. Two hundred (91\%) returned for the second visit and were included in this analysis. The median age of participants was 26 (Interquartile range (IQR), 22-28 years). A quarter (24\%) had no education (median, 4 years [IQR 1-6 years]), and less than half (42.5\%) were married or cohabitating with a partner. Median number of years working in transactional sex was 3.6 years (IQR, 2-6 years); most $(74 \%)$ currently worked in entertainment and service settings. The median number of sex partners in the past 30 days reported was 4 (IQR, 2-10.5 partners) (Table 1).

All participants provided urine samples for ATS testing; 14\% (95\% CI, 9.2, 18.9) were positive, indicating use in the past 48 hours. The prevalence of self-reported ATS use for the same detection period was 15.5\% (95\% CI, 10.4, 20.6).

\section{Sensitivity of self-report}

The sensitivity of self-report was high: $89.3 \%$ (95\% CI, $77.8,100)$ of women who screened positive on the urine

Table 1 Selected sociodemographic and sexual risk characteristics of women participating in the Young Women's Health Study-2

\begin{tabular}{|c|c|c|}
\hline Characteristic & $\mathbf{n}$ & $\%$ \\
\hline \multicolumn{3}{|l|}{ Age } \\
\hline $16-18$ & 11 & 5.5 \\
\hline $19-24$ & 62 & 31.0 \\
\hline $25-29$ & 127 & 63.5 \\
\hline \multicolumn{3}{|c|}{ Years of schooling (0-12 years) } \\
\hline No Education & 48 & 24.0 \\
\hline Primary ( $1-6$ years) & 114 & 57.0 \\
\hline Secondary ( $\geq 7$ years) & 38 & 19.0 \\
\hline \multicolumn{3}{|l|}{ Marital status } \\
\hline Married/Living together & 85 & 42.5 \\
\hline \multicolumn{3}{|c|}{ Divorced/separated/widowed } \\
\hline Single & 34 & 17.0 \\
\hline \multicolumn{3}{|l|}{ Years in transactional sex } \\
\hline$\leq 5$ years & 138 & 69.0 \\
\hline $6-10$ years & 53 & 26.5 \\
\hline$\geq 10$ years & 9 & 4.5 \\
\hline \multicolumn{3}{|l|}{ Type of sex venue } \\
\hline Entertainment/other & 147 & 73.5 \\
\hline Brothels/freelance & 53 & 26.5 \\
\hline \multicolumn{3}{|c|}{ Number of sex partners within last 30 days } \\
\hline$\leq 10$ partners & 150 & 75.0 \\
\hline 11-29 partners & 48 & 24.0 \\
\hline$\geq 30$ partners & 2 & 1.0 \\
\hline
\end{tabular}


toxicology reported recent use of ATS in the same detection period (Table 2). Sensitivity of self-reported ATS use was lower (71.4\%) in women aged 19-24 years compared with the other two groups $(100 \%$ in women under 19 years and $95 \%$ in women over 24 years). Women who worked in the entertainment/service sector had a lower sensitivity of self-reported ATS use (60\%) than those working freelance and/or in brothels (95.7\%) (Table 3c). Sensitivity did not vary by HIV status (Table 3 ).

\section{Specificity of self-report}

Specificity of self-report was high overall: 96.5\% (95\% CI, 93.8, 99.2) of women who had negative ATS urinalysis reported no ATS use (Table 2). Table 3 shows measures of specificity by age, HIV status, and sex-work setting. Specificity of self-report was lowest among very young women $(<19$ years) $(88.9 \%)$ compared with women in the older age groups $(94.6 \%$ and $98.2 \%$ among women age 19-24 years and 25-29 years, respectively); among HIV-positive women (91.3\%) compared with HIVnegative women (97.3\%); and among women working freelance and/or in brothels (92.1\%) compared with women working in the entertainment sector (97.8\%).

\section{Positive predictive value of self-report}

Among 31 women who reported using ATS use within the last 48 hours, $80.6 \%$ had positive urine toxicology tests $(95 \% \mathrm{CI}, 66.7,94.5)$. When analyzed by age group, the youngest groups (ages $<19$ years and 19-24 years) had lower positive predictive value (PPV) (62.5 and $66.7 \%$, respectively) compared with the oldest age group (90\%). Women who worked in the entertainment sector had lower PPV (50\%) of self-report compared with those who worked in freelance or brothel settings (88\%). Participants' HIV status did not appear to affect PPV (Table 3).

\begin{tabular}{|c|c|c|c|}
\hline & & \multicolumn{2}{|c|}{ Urine toxicology result ( $\mathrm{n}$} \\
\hline & & Positive & Negative \\
\hline \multirow[t]{3}{*}{ Self-reported use } & Yes & 25 & 6 \\
\hline & No & 3 & 166 \\
\hline & Percentage & & $95 \% \mathrm{Cl}$ \\
\hline Sensitivity & 89.3 & & $77.8-100$ \\
\hline Specificity & 96.5 & & $93.8-99.2$ \\
\hline Positive predictive value & 80.6 & & $66.7-94.5$ \\
\hline Negative predictive value & 98.2 & & $96.2-100$ \\
\hline
\end{tabular}

Table 3 Self-reported amphetamine-type stimulant use compared with urine toxicology results by age, HIV status, and sex-work venue

Self-reported ATS use compared to urine toxicology results by age group

Urine toxicology results ( $\mathrm{n}$ ) Positive Negative

Age 16-18 years

Self-reported use (n)

Sensitivity (\%)

Specificity (\%)

Positive predictive value (\%)

Negative predictive value (\%)

Age 19-24 years

Self-reported use (n)

No

Sensitivity (\%)

Specificity (\%)

Positive predictive value (\%)

Negative predictive value (\%)

Age 25-29 years

Self-reported use (n)

Sensitivity (\%)

Specificity (\%)

Positive predictive value (\%)

Negative predictive value (\%)
Yes

No

100

88.9

66.7

100

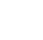

Self-reported ATS use compared to urine toxicology results by HIV status

HIV positive

Self-reported use (n)

Sensitivity (\%)

Specificity (\%)

Positive predictive value (\%)

Negative predictive value (\%)

HIV negative

Self-reported use (n)
Urine toxicology results (n)

Positive Negative

No 1

$95 \% \mathrm{Cl}$

$71.4-100$

$79.8-100$

$59.0-100$

86.8-100

Urine toxicology results (n) Positive Negative

Yes $16 \quad 4$

No $2 \quad 145$

Yes 92


Table 3 Self-reported amphetamine-type stimulant use compared with urine toxicology results by age, HIV status, and sex-work venue (Continued)

\begin{tabular}{lll}
\hline & & $\mathbf{9 5 \%} \mathbf{C l}$ \\
Sensitivity (\%) & 88.9 & $74.4-100$ \\
Specificity (\%) & 97.3 & $94.7-99.9$ \\
Positive predictive value (\%) & 80.0 & $62.5-97.5$ \\
Negative predictive value (\%) & 98.6 & $96.8-100$
\end{tabular}

Self-reported ATS use compared to urine toxicology results by type of sex work setting

Entertainment and/or service

Seff-reported use (n)

Sensitivity (\%)
Specificity (\%)
Positive predictive value (\%)
Negative predictive value (\%)

Freelance and/or brothel

Self-reported use (n)

\begin{tabular}{lll} 
& No & \multicolumn{1}{l}{35} \\
& & $\mathbf{9 5 \%} \mathbf{C l}$ \\
Sensitivity (\%) & 95.7 & $87.3-100$ \\
Specificity (\%) & 92.1 & $82.5-100$ \\
Positive predictive value (\%) & 88.0 & $75.2-100$ \\
Negative predictive value (\%) & 97.2 & $91.9-100$ \\
\hline
\end{tabular}

Negative predictive value of self-report

Overall, the negative predictive value (NPV) of selfreported ATS use was high: $98.2 \%$ (95\% CI, 96.2, 100) of women who reported no ATS use in the last 48 hours had negative urine toxicology tests (Table 2). These results were consistent by age group, HIV status, and sex work venue (Table 3 ).

\section{Discussion}

Overall, results suggest high validity of self-reported ATS use among FSW when compared with urine toxicology screening. In almost all cases (98.2\%) where women reported no ATS use in the past two days, negative urinalysis corroborated self-report. The majority of participants $(89.3 \%)$ with positive urine tests reported ATS use during the same detection period. However, only $81 \%$ of participants $(25 / 31)$ who reported ATS use had positive urine tests. One possible explanation of the low positive predictive value is that women in the study actually used ATS but in such a small quantity that the urine tests failed to detect it. Since ATS is illegal and its purity is unknown, some women could have used the less pure forms of ATS, which may not have been potent enough to be detected by urine testing. The NACD has reported that, among 151 pill samples of ATS tested, $25 \%$ of the samples had purities below 10\% [21]. Although the proportion of women self-reporting ATS use was slightly higher $(15.5 \%)$ than the urine test results (14\%), these rates are not inconsistent and are near perfect. Other studies have documented higher selfreported use compared with urinalysis results, leading to recommendations that multiple methods be used to assess drug use exposures $[27,37,38]$. The high concordance between self-report and test results are suggestive of high internal validity of self-report of ATS in our study population.

Some differences were seen in the performance of selfreport compared with urinalysis when examined by age, HIV status, and sex-work setting. Most notably, there was lower precision between positive self-report and urinalysis tests among younger women and among women working in entertainment or service settings. The lower PPV may relate to lower prevalence of ATS use among these subgroups. We have previously shown that women working in entertainment and service sectors in Cambodia are less likely to use ATS than women working in brothels (17.7\% compared with 35.6\%) [9]. Prevalence of ATS among younger women is slightly lower but not significantly so [9]. Importantly, specificity was high overall, with subgroup analyses showing valid self-report of no ATS use in our sample. This is important for further studies of ATS exposure in this population, for public health surveillance, and potentially for intervention and implementation of drug prevention programs.

The high validity of self-report may be associated with several factors. The women in this study were not reluctant to answer the survey questions or to take the test, as indicated by the high participation rate. This could be due, at least in part, to the fact that the participants were recruited by a known and trusted community-based agent, our collaborating partner (the CWDA), and were comfortable with the staff involved in data collection. Moreover, the women in the study knew that providing truthful responses about their drug use would not result in negative consequences or punitive action.

This study had several limitations. Due to the small sample size and nonsystematic sampling, our estimates lack precision and results may not be representative of all young women engaged in sex work in Phnom Penh or Cambodia. This is particularly true for the stratified analyses, where cell sizes were very small in some cases and prevalence of ATS was lower. Poor recall may have contributed to some discordance, including the relatively low PPV found overall. Approximately one in five 
women (19\%) incorrectly reported recent ATS use. Recall of ATS use could be affected by recent ATS use and its side effects, including sleep deprivation and confusion. It is unknown if this would result in over- or underestimating of self-report. Since women were all informed about the testing as part of the informed consent process and ongoing study-procedure education, some women may have overreported use for the periods about which they were queried. Moreover, urine toxicology tests are not perfectly accurate [35]. Although the urinalysis test is widely accepted as a "gold standard" for substance use validation [39], exclusive reliance on such results does not necessarily improve validity because of problems with false negatives [40]. Many studies comparing self-report, urine, and hair testing results suggest that hair analyses provide higher rates of recent drug use than can be detected by either urine tests or selfreports [27]. Various authors suggest multi-modal testing for the most accurate results $[27,38,41]$.

Despite these limitations, our results suggest a high level of concordance between self-reported ATS use and urine toxicology results in this group of women. Results indicate high prevalence (14\%) of ATS use among FSW, who are also at elevated risk of HIV and other sexually transmitted infections $[3,9]$. There are few, if any, community-based options for ATS users in Cambodia. The finding that selfreport, especially specificity, is valid among young FSW is important because of potential utility in surveillance as well as drug prevention and intervention programs in this population. There is a significant need for evidence-based prevention and drug treatment resources in Cambodia, including potentially cognitive behavioral therapy, contingency management, and possibly new pharmacotherapies to reduce ATS use [42-47]. The forthright self-reporting of drug use by women participating in this study shows that, in a safe and nonpunitive setting, disclosure of accurate drug use is possible. These findings, which are consistent with other studies showing high validity of self-reported drug use, may also be relevant to other vulnerable populations in Cambodia reported to have high rates of ATS use and who may also be in need of interventions, including children, young adults, and men who have sex with men [20]. Indeed, with escalating manufacture and use of ATS throughout Southeast and East Asia, and in consideration of the need for expanded surveillance of drug use to more accurately inform public health and policy responses, selfreported use may be a reliable data collection method. For surveillance, research, and health-care settings, it is important that providers and others address drug-related health issues in a nondiscriminatory manner and without punitive consequences in order to accurately assess and effectively address health and safety issues in high-risk populations.

\section{Competing interests}

The authors declare that they have no competing interests.

\section{Authors' contributions}

All authors contributed to this study: VK, participated in the study design, the primary data analysis and interpretation, and drafted the manuscript; JE contributed to the study conception and design, statistical analysis and interpretation, drafting and review of the manuscript; NS, ES, and M-CC, contributed to the conception and implementation of the study, participated in data collection, and reviewed and provided feedback on the manuscript; LM contributed to the conception and design of the study, and reviewed and edited the manuscript; KP conceived of the study, oversaw data collection and implementation, reviewed and edited the final manuscript. All authors read and approved the final manuscript.

\section{Funding}

This study was financially supported by the research grants from the $\mathrm{NIH}$ (grants 1R21 DA025441 and 1R01NR010995). Dr. Vannda conducted this work in partial fulfillment of the requirements of his MPH degree at UCB and received support from the NIH Fogarty International Center (grant D43 TW00003). Professor Lisa Maher is supported by a National Health and Medical Research Council (NHMRC) Senior Research Fellowship. The Kirby Institute (formerly known as the National Centre in HIV Epidemiology and Clinical Research) is affiliated with the Faculty of Medicine, University of New South Wales, and core-funded by the Australian Government Department of Health and Ageing. Dr. Marie-Claude Couture received financial support for this study from the Canadian Institutes of Health Research (postdoctoral fellowship award).

\section{Acknowledgments}

The authors are gratefully indebted to the women who participated in the study, the diligent effort of site staff, the research teams at the National Center for HIV/AIDS, Dermatology, and STDs, and the Cambodian Women's Development Agency. We also would like to acknowledge the helpful comments of Dr. Art Reingold from University of California at Berkeley (UCB), who was Dr. Vannda's mentor during his time at that institution.

\section{Author details}

${ }^{1}$ School of Public Health, University of California at Berkeley, Berkeley, California, USA. ${ }^{2}$ Department of Epidemiology and Biostatistics, University of California San Francisco, 50 Beale Street, Ste 1200, San Francisco, CA 94105, USA. ${ }^{3}$ Cambodia National Center for HIV, AIDS, Dermatology and STDs, Phnom Penh, Cambodia. ${ }^{4}$ The Kirby Institute (formerly the National Centre in HIV Epidemiology and Clinical Research), Sydney, New South Wales, Australia.

Received: 21 November 2011 Accepted: 21 June 2012

Published: 28 June 2012

\section{References}

1. Mills $E$, Singh S, Orbinski J, Burrows D: The HIV/AIDS epidemic in Cambodia. Lancet Infect Dis 2005, 5(10):596-597.

2. NCHADS: Ministry of Health National Center for HIV, AIDS, Dermatology and STDs.: Annual Report; 2009 [http://www.nchads.org/report.php].

3. Couture MC, Sansothy N, Sapphon V, Phal S, Sichan K, Stein E, Evans J, Maher L, Kaldor J, Vun MC, et al: Young women engaged in sex work in Phnom Penh, Cambodia, have high incidence of HIV and sexually transmitted infections, and amphetamine-type stimulant use: new challenges to HIV prevention and risk. Sex Transm Dis 2010, 38(1):33-39.

4. Sopheab H, Morineau G, Neal JJ, Saphonn V, Fylkesnes K: Sustained high prevalence of sexually transmitted infections among female sex workers in Cambodia: high turnover seriously challenges the $100 \%$ Condom Use Programme. BMC Infect Dis 2008, 8:167.

5. Sopheab H, Saphonn V, Chhea C, Fylkesnes K: Distribution of HIV in Cambodia: findings from the first national population survey. AIDS 2009, 23(11):1389-1395.

6. Charles M: HIV epidemic in Cambodia, one of the poorest countries in Southeast Asia: a success story. Expert Rev Anti Infect Ther 2006, 4(1):1-4.

7. World Health Organization Regional Office for the Western Pacific: Controlling STI and HIV in Cambodia: The success of condom promotion [http://wwwwhoint/hiv/topics/vct/sw_toolkit/controlling_sti_campdf].

8. Sopheab H, Fylkesnes K, Vun MC, O'Farrell N: HIV-related risk behaviors in Cambodia and effects of mobility. J Acquir Immune Defic Syndr 2006, 41 (1):81-86. 
9. Couture MC, Evans $J$, Sothy NS, Stein ES, Sichan K, Maher L, Page K: Correlates of amphetamine-type stimulant use and associations with HIV-related risks among young women engaged in sex work in Phnom Penh, Cambodia. Drug Alcohol Depend 2012, 120(1-3):119-126.

10. Maher L, Phlong P, Mooney-Somers J, Keo S, Stein E, Couture MC, Page K: Amphetamine-type stimulant use and HIV/STI risk behaviour among young female sex workers in Phnom Penh, Cambodia. Int J Drug Policy 2011, 22(3):203-209.

11. Barr AM, Panenka WJ, MacEwan GW, Thornton AE, Lang DJ, Honer WG, Lecomte T: The need for speed: an update on methamphetamine addiction. J Psychiatry Neurosci 2006, 31(5):301-313.

12. McKetin R, Kozel N, Douglas J, Ali R, Vicknasingam B, Lund J, Li JH: The rise of methamphetamine in Southeast and East Asia. Drug Alcohol Rev 2008, 27(3):220-228.

13. Boddiger D: Metamphetamine use linked to rising HIV transmission. Lancet 2005, 365(9466):1217-1218.

14. Buavirat A, Page-Shafer K, van Griensven GJ, Mandel JS, Evans J, Chuaratanaphong J, Chiamwongpat S, Sacks R, Moss A: Risk of prevalent HIV infection associated with incarceration among injecting drug users in Bangkok, Thailand: case-control study. BMJ 2003, 326(7384):308.

15. Cheng WS, Garfein RS, Semple SJ, Strathdee SA, Zians JK, Patterson TL: Differences in sexual risk behaviors among male and female HIVseronegative heterosexual methamphetamine users. Am J Drug Alcohol Abuse 2009, 35(5):295-300.

16. Cheng WS, Garfein RS, Semple SJ, Strathdee SA, Zians JK, Patterson TL: Increased drug use and STI risk with injection drug use among HIV seronegative heterosexual methamphetamine users. J Psychoactive Drugs 2010, 42(1):11-18

17. Colfax G, Shoptaw S: The methamphetamine epidemic: implications for HIV prevention and treatment. Curr HIV/AIDS Rep 2005, 2(4):194-199.

18. Molitor F, Ruiz JD, Flynn N, Mikanda JN, Sun RK, Anderson R: Methamphetamine use and sexual and injection risk behaviors among out-of-treatment injection drug users. Am J Drug Alcohol Abuse 1999, 25 (3):475-493.

19. Wohl AR, Johnson DF, Lu S, Jordan W, Beall G, Currier J, Simon PA: HIV risk behaviors among African American men in Los Angeles County who self-identify as heterosexual. J Acquir Immune Defic Syndr 2002, 31(3):354-360

20. United Nations Office on Drugs and Crime: Patterns and Trends of Amphetamine-Type Stimulants and Other Drugs: Asia and the Pacific. 2010 [http://www.unodc.org/documents/scientific/ATS_Report_2010_web.pdf].

21. National Authority for Combating Drugs: Report on Illicit Drug Data and Routine Surveillance Systems. 2007 [http://www.unodc.org/documents/ eastasiaandpacific/Publications/Projects/NACD_Annual_report2007.pdf]

22. Dillon FR, Turner CW, Robbins MS, Szapocznik J: Concordance among biological, interview, and self-report measures of drug use among African American and Hispanic adolescents referred for drug abuse treatment. Psychol Addict Behav 2005, 19(4):404-413.

23. De Irala J, Bigelow C, McCusker J, Hindin R, Zheng L: Reliability of selfreported human immunodeficiency virus risk behaviors in a residential drug treatment population. Am J Epidemiol 1996, 143(7):725-732

24. Latkin CA, Vlahov D, Anthony JC: Socially desirable responding and selfreported HIV infection risk behaviors among intravenous drug users. Addiction 1993, 88(4):517-526.

25. Des Jarlais DC, Paone D, Milliken J, Turner CF, Miller H, Gribble J, Shi Q Hagan H, Friedman SR: Audio-computer interviewing to measure risk behaviour for HIV among injecting drug users: a quasi-randomised trial. Lancet 1999, 353(9165):1657-1661.

26. Metzger DS, Koblin B, Turner C, Navaline H, Valenti F, Holte S, Gross M, Sheon A, Miller H, Cooley $\mathrm{P}$, et al: Randomized controlled trial of audio computer-assisted self-interviewing: utility and acceptability in longitudinal studies. HIVNET Vaccine Preparedness Study Protocol Team. Am J Epidemiol 2000, 152(2):99-106

27. Harrison L: The validity of self-reported drug use in survey research: an overview and critique of research methods. NIDA Res Monogr 1997, 167:17-36.

28. Fendrich M, Johnson TP: Race/ethnicity differences in the validity of selfreported drug use: results from a household survey. J Urban Health 2005, 82(2 Suppl 3):iii67-iii81.

29. Fendrich $M, X u Y$ : The validity of drug use reports from juvenile arrestees. Int J Addict 1994, 29(8):971-985.
30. Rosay $A B$, Najaka SS, Herz D: Differences in the validity of self-reported drug use across five factors: gender, race, age, type of drug, and offense seriousness. J of Quantitative Criminology 2007, 23(1):41-58.

31. Sherman MF, Bigelow GE: Validity of patients' self-reported drug use as a function of treatment status. Drug Alcohol Depend 1992, 30(1):1-11.

32. Elman I, Krause S, Breiter HC, Gollub RL, Heintges J, Baumgartner WA, Rosen BR, Gastfriend DR: The validity of self-reported drug use in non-treatment seeking individuals with cocaine dependence: correlation with biochemical assays. Am J Addict 2000, 9(3):216-221.

33. Weatherby NL, Needle R, Cesari H, Booth R, McCoy CB, Watters JK, Williams $M$, Chitwood DD: Validity of self-reported drug use among injection drug users and crack cocaine users recruited through street outreach. Eval Program Plann 1994, 17(4):347-355.

34. Hser Yl, Maglione M, Boyle K: Validity of self-report of drug use among STD patients, ER patients, and arrestees. Am J Drug Alcohol Abuse 1999 25(1):81-91

35. Magura S, Kang SY: Validity of self-reported drug use in high risk populations: a meta-analytical review. Subst Use Misuse 1996, 31(9):1131-1153.

36. Hser Yl: Self-reported drug use: results of selected empirical investigations of validity. NIDA Res Monogr 1997, 167:320-343.

37. Cook RF, Bernstein AD, Andrews CM: Assessing drug use in the workplace: a comparison of self-report, urinalysis, and hair analysis. NIDA Res Monogr 1997, 167:247-272

38. Zanis DA, McLellan AT, Randall M: Can you trust patient self-reports of drug use during treatment? Drug Alcohol Depend 1994, 35(2):127-132.

39. Harrison L: The validity of self-reported data on drug use. Journal of Drug Issues 1995, 25(1):91-111.

40. Williams RJ, Nowatzki N: Validity of adolescent self-report of substance use. Subst Use Misuse 2005, 40(3):299-311.

41. Cook RF, Bernstein AD, Arrington TL, Andrews CM, Marshall GA: Methods for assessing drug use prevalence in the workplace: a comparison of self-report, urinalysis, and hair analysis. Int J Addict 1995, 30(4):403-426.

42. Prendergast M, Podus D, Finney J, Greenwell L, Roll J: Contingency management for treatment of substance use disorders: a meta-analysis. Addiction 2006, 101(11):1546-1560.

43. Lee NK, Rawson RA: A systematic review of cognitive and behavioural therapies for methamphetamine dependence. Drug Alcohol Rev 2008, 27 (3):309-317.

44. Rawson RA, Gonzales R, Brethen P: Treatment of methamphetamine use disorders: an update. J Subst Abuse Treat 2002, 23(2):145-150.

45. Rawson RA, Gonzales R, Marinelli-Casey P, Ang A: Methamphetamine dependence: a closer look at treatment response and clinical characteristics associated with route of administration in outpatient treatment. Am J Addict 2007, 16(4):291-299.

46. Shoptaw S, Klausner JD, Reback CJ, Tierney S, Stansell J, Hare CB, Gibson S, Siever M, King WD, Kao U, et al: A public health response to the methamphetamine epidemic: the implementation of contingency management to treat methamphetamine dependence. BMC Publ Health 2006, 6:214

47. Colfax GN, Santos GM, Das M, Santos DM, Matheson T, Gasper J, Shoptaw S, Vittinghoff E: Mirtazapine to reduce methamphetamine use: a randomized controlled trial. Arch Gen Psychiatry 2011, 68(11):1168-1175.

doi:10.1186/1940-0640-7-11

Cite this article as: Kab et al:: Testing for amphetamine-type stimulant (ATS) use to ascertain validity of self-reported ATS use among young female sex workers in Cambodia. Addiction Science \& Clinical Practice 2012 $7: 11$ 